\section{Simplified Modal Expansion to Analyze Frequency-Selective Surfaces: An Equivalent Circuit Approach}

Francisco Mesa, Raúl Rodríguez-Berral, María García-Vigueras, Francisco Medina, and Juan R. Mosig

\begin{abstract}
This communication extends a previously reported analytical circuit that models the plane wave scattering by $2-\mathrm{D}$ arrays of planar patches/apertures embedded in a layered media. In this extension, it is assumed that the current/electric field spatial profile in the patch/aperture region can directly be taken from the eigenfields of a waveguide whose cross section matches the scatterer boundaries. This methodology exploits all the advantages of the circuit modeling and gives place to a very efficient computational tool for many practical frequency-selective surfaces (FSSs). The circuit model is expected to work up to frequencies where the isolated scatterers have their third resonance. Thus, it allows us to provide unified equivalent circuits to deal with conical incidence and with certain symmetric cases involving two or three patches/apertures in the unit cell.
\end{abstract}

Index Terms-Equivalent circuits, electromagnetic scattering by periodic structures, frequency selective surfaces.

\section{INTRODUCTION}

In the microwave and millimeter-wave regimes, two-dimensional (2-D) periodic distributions of metallic patches/apertures (see Fig. 1) are well-known structures widely used for many applications [1]-[4]. A renewed interest in the topic has arisen in connection with extraordinary optical transmission and the birth of metamaterials science [5], [6]. Although the handling of these structures heavily lies on the use of commercial electromagnetic solvers, simple shape scatterers or electrically small unit-cells are amenable to analytical description. Thus, equivalent surface impedances or similar concepts [2], [7] can be used in the latter case. The small electrical size requirement can be removed without losing the analytical (or quasi-analytical) feature by using appropriate equivalent circuits or networks (see, for instance, [8] and references therein or [9]-[11]).

All the methods that yield accurate dynamic circuit models are based on the general idea of transforming the original periodic scattering problem into a generalized waveguide discontinuity problem, since each unit cell can be seen as a generalized waveguide [12] with obstacles or diaphragms inside. The authors have exploited this concept to extract wideband equivalent circuits for several structures

Manuscript received August 25, 2015; revised November 15, 2015; accepted December 19, 2015. Date of publication December 30, 2015; date of current version March 01, 2016. This work was supported in part by the Spanish Ministerio de Economía y Competitividad with European Union FEDER funds (project TEC2013-41913-P) and in part by the Spanish Junta de Andalucía (project P12-TIC-1435).

F. Mesa and R. Rodríguez-Berral are with the Microwaves Group, Department of Applied Physics 1, ETS de Ingeniería Informática, Universidad de Sevilla, Seville, Spain (e-mail: mesa@us.es,rrberral@us.es).

M. García-Vigueras is with the Institut d'Electronique et de Télécommunications de Rennes, INSA de Rennes, Rennes, France (e-mail: Maria.Garcia-Vigueras@insa-rennes.fr).

J. R. Mosig is with the Laboratory of Electromagnetics and Acoustics, Ecole Polytechnique Federale de Laussane, Lausanne, Switzerland.

F. Medina is with the Microwaves Group, Department of Electronics and Electromagnetism, Faculty of Physics, Universidad de Sevilla, Sevilla, Spain (e-mail: medina@us.es).

Color versions of one or more of the figures in this communication are available online at http://ieeexplore.ieee.org.

Digital Object Identifier 10.1109/TAP.2015.2513423
[13]-[15]. In [15], a key fruitful assumption was to consider the spatial distribution of the current/electric field on the scatterer as a frequency-independent function. This idea was extended to 2-D arrays of rectangular patches/apertures in [16] and a seminumerical approach for arbitrarily shaped scatterers was reported in [17]. Although the circuit models in [15]-[17] are accurate over a wide frequency band, they still suffer from the limitation associated with the use of a unique spatial profile in the scatterer [18]. The results worsen when a second intrinsic resonant mode of the patch/slot becomes significant and they are totally wrong when the spatial profile is determined by that second mode. More sophisticated methods, such as the theory of characteristic modes [19], [20], should then be used. In this communication, the authors propose an alternative approach to extend the range of validity of [15]-[17] by using a linear combination of two orthogonal spatial profiles which are taken from the eigenfields of a hollow-pipe waveguide whose cross section has the shape of the scatterer motif. Closed form profiles are available for simple geometries. For more complex patterns, a 2-D eigenvalue problem must be numerically solved (the computational cost of the 2-D problem is almost negligible compared with the usual one required by 3 -D solvers). It is worthy to note that the proposed approach does not yield a general multimodal network model such as the one reported in [16] but a circuit representation of the equivalent discontinuity admittance met by a given incident wave. As a result, the new circuit models are valid over a much wider frequency band that covers the first two intrinsic resonances of the patches/apertures. Interestingly, they can also account for unit cells involving two or three patches or apertures. The analysis of cross-polarization effects is also simplified with respect to other approaches [16].

This communication is organized as follows. In Section II, a circuit model is systematically derived for a planar array of patches. The inclusion of a multilayered environment and the formulation of the aperture problem is briefly discussed. Section III presents a selected group of examples to illustrate the validity, advantages, and limitations of the method. Finally, conclusion is drawn in Section IV.

\section{Derivation of the Equivalent Circuits}

In this section, an equivalent circuit is derived that models the scattering of a plane wave impinging on a periodic array of metallic scatterers embedded in a layered environment, see Fig. 1(a). This problem can be posed in terms of the equivalent waveguide discontinuity scenario (unit cell) depicted in Fig. 1(b). The walls of the generalized waveguide [12] are periodic boundaries. For convenience, it will be first considered the case of semi-infinite homogeneous dielectrics at both sides of the scatterers. In related papers [10], [16], [21], [22], this problem has usually been developed for apertures-like discontinuities and now it will be briefly exposed for the case of obstacles. Thus, assuming a time-harmonic regime ( $\omega$ is the angular frequency), a Floquet analysis of the discontinuity $(z=0)$ as that reported in [16] should be carried out.

\section{A. Patch Discontinuity}

The surface current-density phasor in the patch $\mathbf{J}_{p}(x, y)$ is assumed to be given by the following superposition:

$$
\mathbf{J}_{p}(x, y ; \omega)=A_{1}(\omega) \mathcal{J}_{1}(x, y)+A_{2}(\omega) \mathcal{J}_{2}(x, y)
$$

where $A_{i}(\omega)$ are complex frequency-dependent coefficients and $\mathcal{J}_{i}(x, y)$ are certain given spatial profiles. These profiles can be 


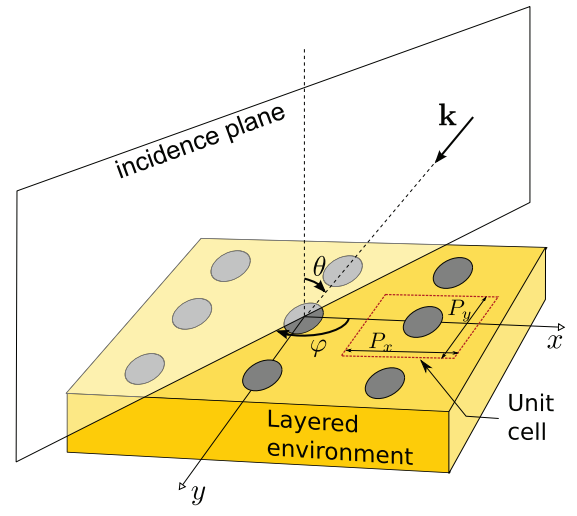

(a)

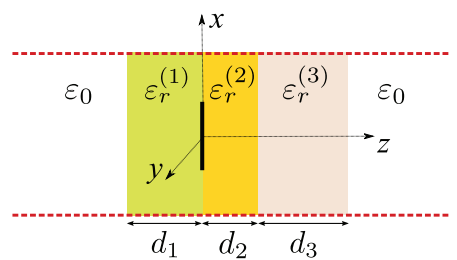

(b)

Fig. 1. (a) 2-D rectangular periodic distribution of scatterers (metallic patches or apertures in the metallic screen) embedded in a layered dielectric medium. (b) Lateral view of a single unit cell for a three-layer structure.

obtained from appropriate physical assumptions [18] or derived from a properly chosen characteristic eigenproblem. In this communication, our eigenproblem of choice is the 2-D modal propagation problem of a hollow metallic waveguide whose cross section is given by the geometry of the patch after invoking the duality principle [23]. With this choice, the obtaining of the spatial profiles only involves the solution of a full-wave 2-D problem. Moreover, for scatterers with canonical geometries, closed-form expressions are available. When complex geometries are involved, we compute the eigenfields by using the so-called "solve ports only" option of HFSS [24], in which HFSS calculates the natural field patterns (or modes) that can exist inside a transmission structure with the same cross-section as the port.

Adopting the notation and the guidelines in [16], if the current density in (1) is made equal to the jump of the magnetic fields in a weak sense [both quantities are equally projected in the unit cell (u.c.)]; namely

$$
\begin{aligned}
& \int_{\text {u.c. }} \mathrm{e}^{\mathrm{j} \mathbf{k}_{\mathrm{t}, q} \cdot \boldsymbol{\rho}} \hat{\mathbf{e}}_{q} \cdot\left\{\hat{\mathbf{z}} \times\left[\mathbf{H}^{(2)}(x, y)-\mathbf{H}^{(1)}(x, y)\right]\right\} \mathrm{d} x \mathrm{~d} y \\
& =\int_{\text {u.c. }} \mathrm{e}^{\mathrm{j} \mathbf{k}_{\mathrm{t}, q} \cdot \boldsymbol{\rho}} \hat{\mathbf{e}}_{q} \cdot \mathbf{J}_{p}(x, y) \mathrm{d} x \mathrm{~d} y
\end{aligned}
$$

the following relations are obtained:

$$
\begin{aligned}
{\left[Y_{0}^{(1)}\left(1-R_{0}\right)-Y_{0}^{(2)}\left(1+R_{0}\right)\right] } & =A_{1} N_{1,0}+A_{2} N_{2,0} \\
-\left[Y_{q}^{(1)}+Y_{q}^{(2)}\right] V_{q} & =A_{1} N_{1, q}+A_{2} N_{2, q} .
\end{aligned}
$$

The $N_{i, q}(i=1,2)$ coefficients were identified as transformer turn ratios in [10], [16], and [21], and are given by

$$
N_{i, q}=\tilde{\mathcal{J}}_{i}\left(\mathbf{k}_{\mathrm{t}, q}\right) \cdot \hat{\mathbf{e}}_{q}
$$

where

$$
\widetilde{\mathcal{J}}_{i}\left(\mathbf{k}_{\mathrm{t}, q}\right)=\int_{\text {u.c. }} \mathcal{J}_{i}(x, y) \mathrm{e}^{\mathrm{j} \mathbf{k}_{\mathrm{t}, q} \cdot \boldsymbol{\rho}} \mathrm{d} x \mathrm{~d} y
$$

is the Fourier transform at $\mathbf{k}_{\mathrm{t}, q}=k_{x n} \hat{\mathbf{x}}+k_{y m} \hat{\mathbf{y}}$ of the $i$ th current spatial profile in the patch. From (4), it is readily obtained that the amplitudes of the harmonics of the electric field are given by

$$
V_{q}=-\frac{A_{1} N_{1, q}+A_{2} N_{2, q}}{Y_{q}^{(1)}+Y_{q}^{(2)}}
$$

which allows us to write $\mathbf{E}(x, y)$ as

$\mathbf{E}(x, y)=\left(1+R_{0}\right) \mathrm{e}^{-\mathrm{j} \mathbf{k}_{\mathrm{t}, 0} \cdot \boldsymbol{\rho}} \hat{\mathbf{e}}_{0}-\sum_{q}^{\prime} \frac{A_{1} N_{1, q}+A_{2} N_{2, q}}{Y_{q}^{(1)}+Y_{q}^{(2)}} \mathrm{e}^{-\mathrm{j} \mathbf{k}_{\mathrm{t}, q} \cdot \boldsymbol{\rho}} \hat{\mathbf{e}}_{q}$.

After imposing that the transverse electric field over the patch is null when projected over each spatial profile

$$
\int_{\text {u.c. }} \mathcal{J}_{i}^{*}(x, y) \cdot \mathbf{E}(x, y) \mathrm{d} x \mathrm{~d} y=0 \quad(i=1,2)
$$

the following pair of equations are obtained:

$$
\begin{aligned}
N_{i, 0}^{*}\left(1+R_{0}\right)= & A_{1} \sum_{q}^{\prime} \frac{N_{1, q}}{Y_{q}^{(1)}+Y_{q}^{(2)}} N_{i, q}^{*} \\
& +A_{2} \sum_{q}^{\prime} \frac{N_{2, q}}{Y_{q}^{(1)}+Y_{q}^{(2)}} N_{i, q}^{*} \quad(i=1,2) .
\end{aligned}
$$

Note that the use of the complex conjugate ${ }^{*}$ is key to ensure the physical consistency of the formulation; namely, power conservation at the discontinuity plane. Now, it is convenient to rewrite the pair of equations in (10) as

$$
V_{0}=Z_{i 1} I_{0,1}+Z_{i 2} I_{0,2} \quad(i=1,2)
$$

where it can be identified that $(i, j=1,2)$

$$
\begin{aligned}
& V_{0}=-\left(1+R_{0}\right) \\
& I_{0, i}=-A_{i} N_{i, 0} \\
& Z_{i j}=\sum_{q}^{\prime} \frac{N_{i, q}^{*} N_{j, q}}{N_{i, 0}^{*} N_{j, 0}} \frac{1}{Y_{q}^{(1)}+Y_{q}^{(2)}} .
\end{aligned}
$$

The system of equations (11) can be solved to obtain $I_{0,1}$ and $I_{0,2}$ [and hence $A_{1}(\omega)$ and $A_{2}(\omega)$ ] as

$$
\begin{aligned}
I_{0,1} & =\frac{Z_{22}-Z_{12}}{Z_{11} Z_{22}-Z_{12} Z_{21}} V_{0} \\
I_{0,2} & =\frac{Z_{11}-Z_{21}}{Z_{11} Z_{22}-Z_{12} Z_{21}} V_{0} .
\end{aligned}
$$

In order to find the physical meaning of $I_{0,1}$ and $I_{0,2}$, first note that the $V_{0}$ coefficient defined above in (12) can be interpreted as the voltage drop associated with the incident harmonic at the discontinuity plane, whereas the quantity

$$
I_{0}=Y_{0}^{(2)}\left(1+R_{0}\right)-Y_{0}^{(1)}\left(1-R_{0}\right)
$$

can be interpreted as the difference between the "currents" associated with the incident harmonic at both sides of the discontinuity. Combining (3) and (13), it can be written as

$$
I_{0}=I_{0,1}+I_{0,2}
$$

which allows to interpret $I_{0,1}$ and $I_{0,2}$ as partial contributions to the discontinuity in the incident mode "current" due to the presence of the obstacle. The above discussion allows us to identify the ratio between 


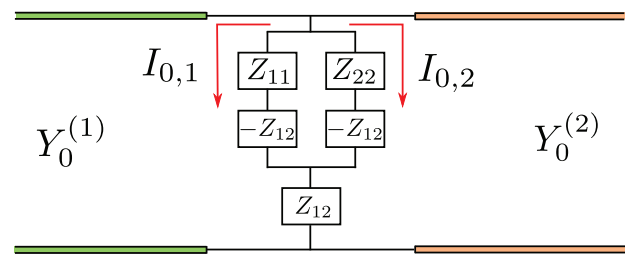

(a)

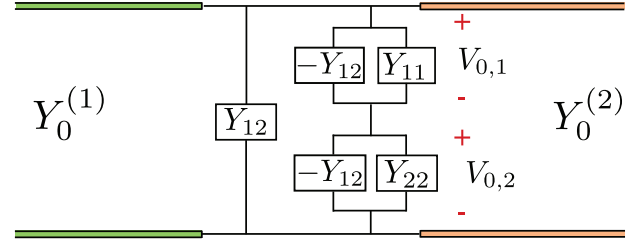

(b)

Fig. 2. Equivalent circuit that models the discontinuity formed by (a) metallic patches and (b) apertures. The model takes into account two independent spatial profiles of the unknown quantity in the discontinuity.

$I_{0}$ and $V_{0}$ as the equivalent admittance of the discontinuity for the incident harmonic. Using (15), (16), and (18), the following equation is obtained:

$$
\frac{I_{0}}{V_{0}} \equiv Y_{\mathrm{eq}}=\frac{\left(Z_{22}-Z_{12}\right)+\left(Z_{11}-Z_{21}\right)}{Z_{11} Z_{22}-Z_{12} Z_{21}} .
$$

Unfortunately, the authors have not been able to find out a simple and general network topology that correspond to (19) in the most general case. However, in those common and practical cases where $Z_{12}=Z_{21}$, the following expression is found for $Z_{\mathrm{eq}}=Y_{\mathrm{eq}}^{-1}$ :

$$
Z_{\mathrm{eq}}=\left[\frac{1}{Z_{11}-Z_{12}}+\frac{1}{Z_{22}-Z_{12}}\right]^{-1}+Z_{12}
$$

whose topology is shown in Fig. 2(a). By subtracting both equations of the pair in (11), it is obtained that

$$
I_{0,1}\left(Z_{11}-Z_{21}\right)=I_{0,2}\left(Z_{22}-Z_{12}\right)
$$

which makes it possible to identify the currents $I_{0,1}$ and $I_{0,2}$ with those drawn in Fig. 2(a).

The equality $Z_{12}=Z_{21}$ is satisfied provided that the factor involving the transformers ratios in (14) is a real number (for instance, if the transformer turn ratios are either real or imaginary). This situation is found when each of the spatial profiles $\mathcal{J}_{i}(x, y)$ does have a given parity (even or odd), which is possible if the layout of the unit cell is symmetrical. Thus, nonsymmetric single scatterers as well as a nonsymmetric distribution of symmetric scatterers will not yield the topological representation for $Z_{\text {eq }}$ shown in Fig. 2(a).

If the structure is lossless and the operation frequency is below the diffraction threshold, then its corresponding equivalent impedance $Z_{\mathrm{eq}}$ must be purely imaginary according to power conservation. This property is satisfied provided that the complex conjugate in the turn ratios in (14) are incorporated in the formulation, since it implies $Z_{21}=-Z_{12}^{*}$ and $\operatorname{Re}\left(Z_{j j}\right)=0(j=1,2)$, which makes that $\operatorname{Re}\left(Z_{\text {eq }}\right)=0$. If the conjugate is ignored (ensuring that $Z_{12}=Z_{21}$ in all cases), it would lead in general to the nonphysical condition $\operatorname{Re}\left(Z_{\text {eq }}\right) \neq 0$.

Regardless of the possible existence of a simple equivalent network topology for $Z_{\mathrm{eq}}$, the knowledge of the equivalent admittance given by (19) allows for the computation of the scattering parameters that relate the incident wave with its corresponding reflected and transmitted waves (namely, with the scattered $q=0$ harmonic). Furthermore, at the light of (7), the following generalized reflection coefficient can readily be obtained that relates the amplitude of the reflected wave of an arbitrary harmonic $q$ with the unit-amplitude of the incident harmonic

$$
S_{11}^{q, 0}=\sqrt{\frac{Y_{q}^{(1)}}{Y_{0}^{(1)}}} V_{q}=-\sqrt{\frac{Y_{q}^{(1)}}{Y_{0}^{(1)}}} \frac{A_{1} N_{1, q}+A_{2} N_{2, q}}{Y_{q}^{(1)}+Y_{q}^{(2)}} .
$$

Although the coupling between the incident mode and a different mode does not have a direct representation in terms of a multimodal network, (22) shows that it is possible to compute the corresponding cross-pol reflection coefficient a posteriori from the circuit elements and the values of the current and voltages obtained after solving the circuit.

\section{B. Aperture Discontinuity}

The complementary situation where the discontinuity is an aperture in a thin conducting wall instead of a metallic planar obstacle can be studied in a similar way as before. This situation was already treated in [10] in a similar manner and here only the final results will be reported for completitude (the use of the conjugate to ensure power conservation is now taken in contrast to [10]). Thus, assuming that the transverse electric field in the aperture can be written as

$$
\mathbf{E}_{a}(x, y ; \omega)=A_{1}(\omega) \mathcal{E}_{1}(x, y)+A_{2}(\omega) \mathcal{E}_{2}(x, y)
$$

the following system of equations is obtained:

$$
I_{0}=Y_{i 1} V_{0,1}+Y_{i 2} V_{0,2} \quad(i=1,2)
$$

where $I_{0}$ was already defined in (17) and

$$
\begin{aligned}
& V_{0, i}=A_{i} N_{i, 0} \\
& Y_{i j}=\sum_{q} \frac{N_{i, q}^{*} N_{j, q}}{N_{i, 0}^{*} N_{j, 0}}\left[Y_{q}^{(1)}+Y_{q}^{(2)}\right]
\end{aligned}
$$

with the transformer ratios now given by

$$
N_{j, q}=\widetilde{\mathcal{E}}_{j}\left(\mathbf{k}_{\mathrm{t}, q}\right) \cdot \hat{\mathbf{e}}_{q} .
$$

As it happened for the patch-like discontinuity, only when $Y_{12}=Y_{21}$, it has been possible to find the topology shown in Fig. 2(b) for the equivalent admittance of the aperture discontinuity

$$
Y_{\mathrm{eq}}=\left[\frac{1}{Y_{11}-Y_{12}}+\frac{1}{Y_{22}-Y_{12}}\right]^{-1}+Y_{12} .
$$

It is worthy to note here that the physical meaning of the partial voltages shown in Fig. 2(b) is actually very appealing when there are two apertures in the unit cell. In that case, $V_{0, i}$ can be associated with the voltage drop across the $i$ th-aperture, characterized by an admittance $Y_{i i}-Y_{12}$, and with the branch having the admittance $Y_{12}$ accounting for the coupling between apertures. This interpretation correlates perfectly with the physical discussions given by some of the authors in [14] for the case of compound gratings. In that paper, the proposed equivalent circuit that modeled the compound grating structure was heuristically derived from a physical rationale. Now, the mathematical basis for this model is given, and it also suggests how to find out the way to extend the present procedure to deal also with apertures of nonnegligible thickness.

In order to obtain the scattering parameters that relate different modes, it should be noted that the amplitude $V_{q}$ of the electric field is found to be

$$
V_{q}=A_{1} N_{1, q}+A_{2} N_{2, q}
$$


and, therefore, the general cross-pol reflection coefficient can readily be obtained as

$$
S_{11}^{0, q}=\sqrt{\frac{Y_{q}^{(1)}}{Y_{0}^{(1)}}}\left(A_{1} N_{1, q}+A_{2} N_{2, q}\right) .
$$

\section{Other Considerations}

As discussed in [16], the presence of a layered environment to the left/right of the aperture/patch discontinuity is simply taken into account by replacing the $Y_{q}^{(1 / 2)}$ admittances of each harmonic with the corresponding input admittances seen to the left $(\mathrm{L}) / \operatorname{right}(\mathrm{R})$ of the aperture by this harmonic, i.e.,

$$
Y_{q}^{(1 / 2)} \rightarrow Y_{\mathrm{in}, q}^{(\mathrm{L} / \mathrm{R})}
$$

With respect to the numerical computation of $Z_{i j}$ in (14) as well as $Y_{i j}$ in (26), the discussion about localized and propagative modes/harmonics given, for instance, in [16] and [22] should be considered. It means that, in a frequency sweep, only the explicit summation of very few terms in the infinite series has to be carried out for each frequency point. The remaining part of the series can be identified with regular capacitance/inductance elements that are frequency independent, and that have to be computed only once.

\section{NUMERICAL RESULTS AND DISCUSSIONS}

\section{A. Array of Single Multiresonant Rectangular Scatterers}

A first case to validate our circuit-model approach is the widely studied array of rectangular patches printed on a dielectric slab [1], [25]. In [16] and [17], it was discussed that the circuit-model approach proposed by the authors (a single spatial profile was assumed) was certainly of wideband nature and that its limit of validity was imposed by the presence of more than one resonance in the scatterer susceptible to be excited by the impinging wave. In the case of normal incidence and rectangular patches, this limit was found to be the third intrinsic resonance of the dipole [17] (the second odd-symmetry resonance of the metallic patch is not excited by the normally incident plane wave). However, if the incidence is oblique/conical, the second odd resonance can certainly be excited, thus lowering the limit of validity of the circuit model. This fact was widely discussed in connection with [16, Fig. 10], which clearly showed that the use of a single spatial profile could account for either the first or the second resonance of the metallic dipole but not for both of them. This same structure is now revisited and the results of the equivalent circuit proposed in this communication are shown in Fig. 3. Our results have been computed using the following even and odd spatial profiles (taken directly from the first and second modes of the associated rectangular waveguide):

$$
\mathcal{J}_{n}(x, y)=\cos \left(\frac{n \pi x}{w_{x}}\right) \operatorname{rect}\left(\frac{x}{w_{x}}\right) \operatorname{rect}\left(\frac{y}{w_{y}}\right) \hat{\mathbf{x}}
$$

where $n=1,2$ and $\operatorname{rect}(x)$ is the rectangular function.

It should be noted that the present approach has allowed us to study the frequency behavior of this array of patches under oblique incidence up to frequencies well inside the diffraction regime and that the comparison of our results with those provided by HFSS [24] is excellent. Furthermore, the numerical effort required by the present quasianalytical methodology is almost negligible (less than $1 \mathrm{~s}$ for 1000 frequency points) in comparison with that required by commercial electromagnetic simulators. Also, it should be pointed out that any resonance of the structure associated with reflections/transmissions in the

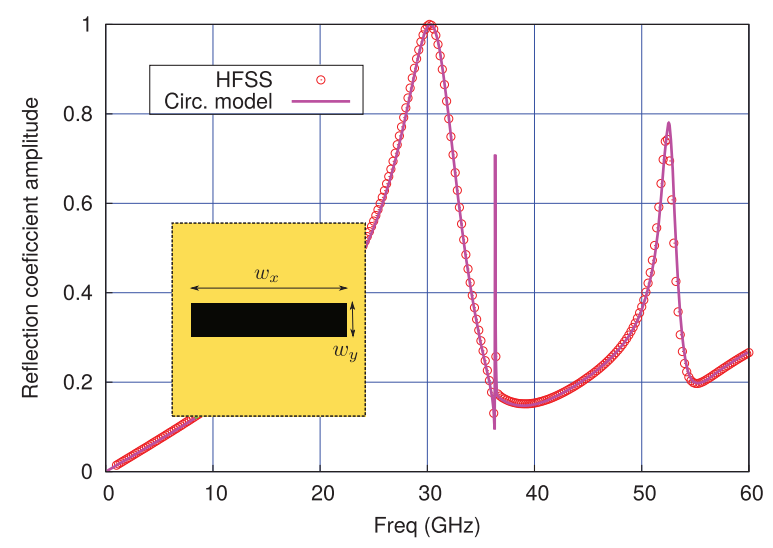

Fig. 3. Magnitude of the reflection coefficient under oblique TM-incidence in the $x z$-plane $\left(\varphi=0^{\circ}, \theta=40^{\circ}\right)$ for the same structure studied in [16, Fig. 10]. Following the notation employed in Fig. 1(b) [distances in $\mathrm{mm}$ ]: $\varepsilon_{r}^{(2)}=3$, $d_{1}=0, d_{2}=0.5, d_{3}=0, P_{x}=P_{y}=5, w_{x}=3.5$, and $w_{y}=0.5$.

layered environment is rigorously accounted for by the methodology employed in this communication. Thus, the only resonances that can eventually limit the applicability of the present approach are those related to the individual scatterers.

\section{B. Array of Two/Three Rectangular Scatterers Per Unit Cell}

If the unit cell of the periodic array under analysis has two patches/apertures and the frequency band of interest is below the second excitable resonance of each individual scatterer, then the spatial profile in the scatterers (patches/apertures) can be written, in general, as

$$
\begin{aligned}
\mathbf{F}(\boldsymbol{\rho} ; \omega) & =A_{1}(\omega) \mathcal{F}_{1}(\boldsymbol{\rho})+A_{2}(\omega) \mathcal{F}_{2}(\boldsymbol{\rho}) \\
& =A_{1}(\omega) \mathbf{F}_{1}\left(\boldsymbol{\rho}-\boldsymbol{\rho}_{\mathrm{c}, 1}\right)+A_{2}(\omega) \mathbf{F}_{2}\left(\boldsymbol{\rho}-\boldsymbol{\rho}_{\mathrm{c}, 2}\right)
\end{aligned}
$$

where $\boldsymbol{\rho}_{\mathrm{c}, j}$ is the position of the center of scatterer $j(j=1,2)$ and $\mathbf{F}_{j}(\boldsymbol{\rho})$ is the assumed spatial profile when the scatterer $j$ is centered in the unit cell. Since the transformer ratios are actually related to the Fourier transforms of the spatial profiles, it can be noted that

$$
\widetilde{\mathcal{F}}_{j}\left(\mathbf{k}_{\mathrm{t}, q}\right)=\widetilde{\mathbf{F}}_{j}\left(\mathbf{k}_{\mathrm{t}, q}\right) \mathrm{e}^{-\mathrm{j} \mathbf{k}_{\mathrm{t}, q} \cdot \boldsymbol{\rho}_{\mathrm{c}, j}}
$$

Therefore, this simple consideration allows for the treatment of two scatterers per unit cell under the reported given assumptions. Our results for this situation are compared in Fig. 4(a) with the results obtained with HFSS showing an excellent agreement in the complete nondiffractive frequency regime.

With a similar strategy as before, it is also possible to study the case of three scatterers per unit cell as long as the symmetry of the unit cell allows us to write the unknown current (electric field) in the patches (apertures) in the following way:

$$
\begin{aligned}
\mathbf{F}(\boldsymbol{\rho} ; \omega)= & A_{1}(\omega) \mathbf{F}_{1}\left(\boldsymbol{\rho}-\boldsymbol{\rho}_{\mathrm{c}, 1}\right) \\
& +A_{2}(\omega)\left[\mathbf{F}_{2}\left(-\boldsymbol{\rho}-\boldsymbol{\rho}_{\mathrm{c}, 2}\right)+\mathbf{F}_{2}\left(\boldsymbol{\rho}-\boldsymbol{\rho}_{\mathrm{c}, 2}\right)\right] .
\end{aligned}
$$

Using these profiles, our results for an array with three dipoles per unit cell is shown in Fig. 4(b). The agreement of our results with those obtained from HFSS is very satisfactory. Again, it is important to point out that the computation effort of our quasi-analytical approach in the above two cases is negligible in comparison with that required by commercial electromagnetic simulators. 


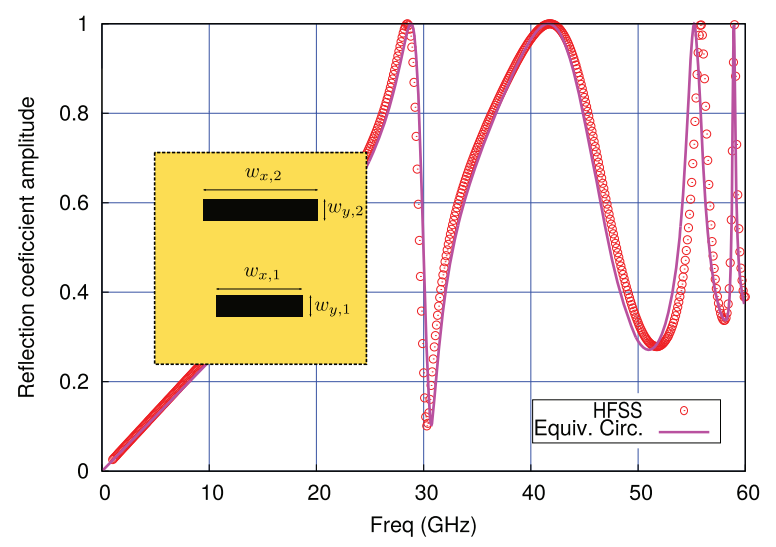

(a)

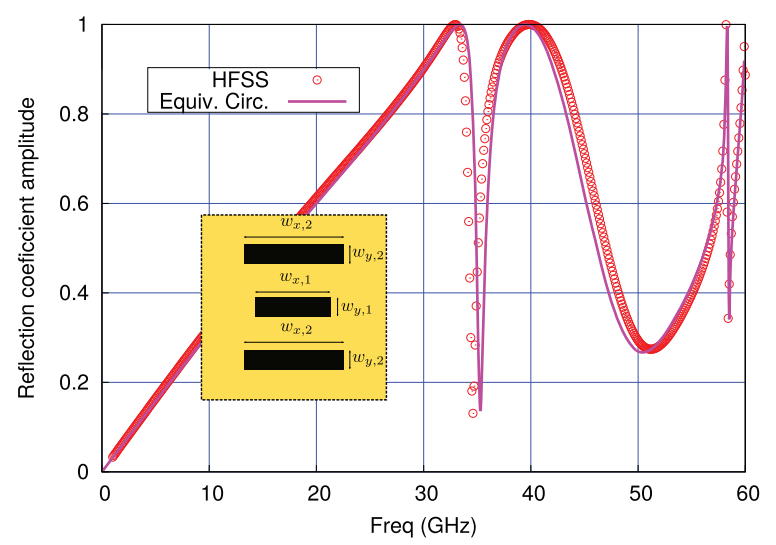

(b)

Fig. 4. Magnitude of the reflection coefficient under normal TM incidence in the $x z$-plane $\left(\varphi=0^{\circ}\right)$ for an FSS whose unit cell has (a) two dipoles and (b) three dipoles per unit cell with the following characteristics (distances in $\mathrm{mm}): \varepsilon_{r}^{(2)}=3.0, d_{1}=0, d_{2}=0.5, d_{3}=0, P_{x}=P_{y}=5$, and $w_{x, 1}=2.5, w_{y, 1}=0.5, w_{x, 2}=3.5, w_{y, 2}=0.5$. (a) $\boldsymbol{\rho}_{\mathrm{c}, 1}=(0,-1)$, $\boldsymbol{\rho}_{\mathrm{c}, 2}=(0,1)$. (b) $\boldsymbol{\rho}_{\mathrm{c}, 1}=(0,0), \boldsymbol{\rho}_{\mathrm{c}, 2}=(0,1)$.

\section{Conical Incidence}

The so-called conical incidence appears when the incidence plane of the impinging wave does not coincide with any of the principal planes of the structure $(\varphi \neq 0, \pi / 2, \pi, 3 \pi / 2)$ and the wave incidence is not normal $(\theta \neq 0)$. This situation is considerably more involved than the case of oblique incidence along principal planes. Some of the authors of this communication dealt with this situation by means of a superposition of four auxiliary problems in [16]. Now, with the proposed use of two spatial profiles, it is possible to study the case of conical incidence by expressing the unknown current (electric field) in the patch (aperture) as

$$
\mathbf{F}(\boldsymbol{\rho} ; \omega)=A_{1}(\omega) \mathcal{F}_{x}(\boldsymbol{\rho}) \hat{\mathbf{x}}+A_{2}(\omega) \mathcal{F}_{y}(\boldsymbol{\rho}) \hat{\mathbf{y}}
$$

where $\mathcal{F}_{x}(\boldsymbol{\rho})$ and $\mathcal{F}_{y}(\boldsymbol{\rho})$ are the assumed single spatial profiles associated with problems whose incidence is along the principal planes of the structure (namely, when the incident electric field is directed along the axes $x$ or $y$ ). Thus, for the case of a rectangular patch/aperture, these functions can be written as $(\alpha=x, y)$

$$
\mathcal{F}_{\alpha}(\boldsymbol{\rho})=\cos \left(\frac{\pi \alpha}{w_{\alpha}}\right) \operatorname{rect}\left(\frac{x}{w_{x}}\right) \operatorname{rect}\left(\frac{y}{w_{y}}\right) \text {. }
$$

The above assumption implies that the corresponding frequency limit of validity will be determined by the appearance of the second

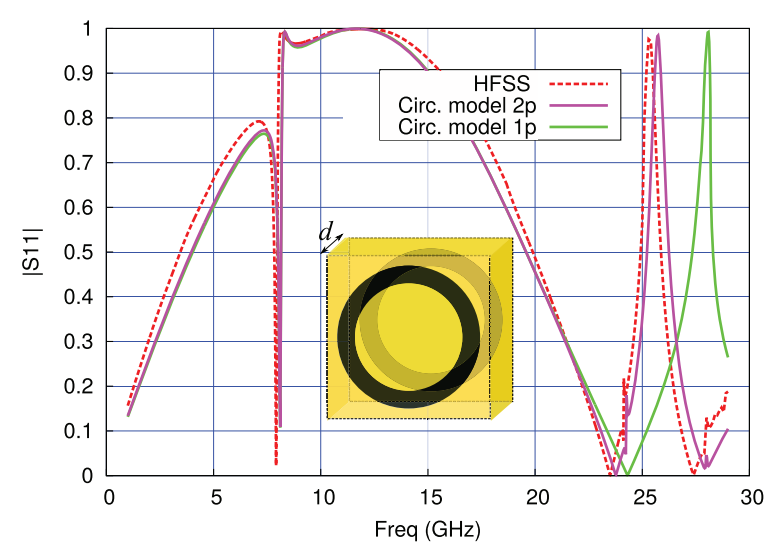

Fig. 5. Reflection coefficient of a pair of coupled circular-ring FSS's printed on a dielectric slab of thickness $d=1 \mathrm{~mm}$ and $\varepsilon_{r}=2.2, \tan \delta=9 \times 10^{-4}$ under normal incidence. The external radius of the patch is $R_{e}=4.82 \mathrm{~mm}$ and the inner radius is $R_{i}=3.82 \mathrm{~mm} . P_{x}=P_{y}=10.64 \mathrm{~mm}$.

excitable resonance of the scatterer. The same case already studied in [16], where the cross-pol reflection coefficient was computed, has been recomputed with the present methodology (although not explicitly shown here), and the agreement with HFSS results is even better than in [16].

\section{Array of Multiresonant Scatterers of Arbitrary Geometry}

Next, it is considered the case of a periodic array of scatterers with arbitrary geometry (no closed-form expressions for the spatial profiles are generally available). Thus, Fig. 5 shows a comparison between our results and those provided by HFSS for a pair of coupled circular-ring FSS's. The coupled structure has been solved by applying the evenodd excitation technique [16], [23] in such a way that the same two first excitable eigenfields extracted from the HFSS eigensolver tool (which requires a very short computation time) have been repetitively used in all the necessary computations. Our results in Fig. 5 are plotted for two cases: 1) only the fundamental eigenfield is used in the expansion (1p); 2) the fundamental and the first high-order eigenfields are employed (2p). The comparison of the corresponding curves clearly shows that the introduction of the second spatial profile in the analysis considerably extends the range of application of the circuit model approach, since the addition of the second spatial profile makes that the second resonance of the individual scatterer is better captured. Our data computed with two spatial profiles show a reasonably good agreement with HFSS in the complete nondiffractive regime.

\section{CONCLUSION}

Two new equivalent circuit formulations are developed in this communication to account for the plane wave scattering of planar structures consisting of two-dimensional periodic arrays of metal patches or apertures made in thin metal films. These periodic metal grids are assumed to be printed on (or embedded in) a multilayered dielectric medium. For highly symmetric unit cells, the formulations yield specific realizable circuit topologies consisting of series and shunt connections of quasi-analytically known impedances or admittances, which add physical insight to the understanding of the periodic structure operation. Asymmetrical cells do not result in simple specific circuit topologies but quasi-analytical expressions for the discontinuity admittance or impedance are still obtained. The derivation of the equivalent circuits is based on the use of a linear combination of two 
orthogonal spatial profiles. These approximate profiles are obtained from the solution of the 2-D eigenvalue problem associated with a hollowpipe waveguide whose cross section is the geometry of the scatterer. The present formulation yields equivalent circuits for a chosen pair of input/output modes with a much wider frequency band than most available equivalent circuit formulations. The virtues and drawbacks of the formulation have been illustrated through a set of selected examples.

\section{REFERENCES}

[1] B. Munk, Frequency Selective Surfaces: Theory and Design. Hoboken, NJ, USA: Wiley, 2000.

[2] O. Luukkonen et al., "Simple and accurate analytical model of planar grids and high-impedance surfaces comprising metal strips or patches," IEEE Trans. Antennas Propag., vol. 56, no. 6, pp. 1624-1632, Jun. 2008.

[3] M. Al-Joumayly and N. Behdad, "A new technique for design of low-profile, second-order, bandpass frequency selective surfaces," IEEE Trans. Antennas Propag., vol. 57, no. 2, pp. 452-459, Feb. 2009.

[4] D. Cavallo, W. H. Syed, and A. Neto, "Closed-form analysis of artificial dielectric layers-Parts I-II: Properties of a single layer under plane-wave incidence," IEEE Trans. Antennas Propag., vol. 62, no. 12, pp. 62566273, Dec. 2014.

[5] T. W. Ebbesen, H. J. Lezec, H. F. Ghaemi, T. Thio, and P. A. Wolff, "Extraordinary optical transmission through sub-wavelength hole arrays," Nature, vol. 391, pp. 667-669, Feb. 1998.

[6] F. Capolino, Ed., Metamaterial Handbook: Theory and Phenomena of Metamaterials. Boca Raton, FL, USA: CRC Press, 2009.

[7] A. B. Yakovlev, Y. R. Padooru, G. W. Hanson, A. Mafi, and S. Karbasi, "A generalized additional boundary condition for mushroom-type and bedof-nails-type wire media," IEEE Trans. Microw. Theory Techn., vol. 59, no. 3, pp. 527-531, Mar. 2011.

[8] F. Costa, A. Monorchio, and G. Manara, "An overview of equivalent circuit modeling techniques of frequency selective surfaces and metasurfaces," Appl. Comput. Electromagn. Soc. J., vol. 29, no. 12, pp. 960-976, Dec. 2014.

[9] M. Guglielmi and A. A. Oliner, "Multimode network description of a planar periodic metal-strip grating at a dielectric interface-Part I: Rigorous network formulations," IEEE Trans. Microw. Theory Techn., vol. 37, no. 3, pp. 535-541, Mar. 1989.

[10] R. Dubrovka, J. Vazquez, C. Parini, and D. Moore, "Multi-frequency and multi-layer frequency selective surface analysis using modal decomposition equivalent circuit method," IET Microw. Antennas Propag., vol. 3, no. 3, pp. 492-500, Mar. 2009.

[11] B. Blázquez, N. Llombart, D. Cavallo, A. Freni, and A. Neto, "A rigorous equivalent network for linearly polarized THz absorbers," IEEE Trans. Antennas Propag., vol. 62, no. 10, pp. 5077-5088, Oct. 2014.

[12] J. E. Varela and J. Esteban, "Characterization of waveguides with a combination of conductor and periodic boundary contours: Application to the analysis of bi-periodic structures," IEEE Trans. Microw. Theory Techn., vol. 60, no. 3, pp. 419-430, Mar. 2012.

[13] F. Medina, F. Mesa, and R. Marqués, "Extraordinary transmission through arrays of electrically small holes from a circuit theory perspective," IEEE Trans. Microw. Theory Techn., vol. 56, no. 12, pp. 3108-3120, Dec. 2008.

[14] F. Medina, F. Mesa, and D. C. Skigin, "Extraordinary transmission through arrays of slits: A circuit theory model," IEEE Trans. Microw. Theory Techn., vol. 58, no. 1, pp. 105-115, Jan. 2010.

[15] R. Rodríguez-Berral, C. Molero, F. Medina, and F. Mesa, "Analytical wideband model for strip/slit gratings loaded with dielectric slabs," IEEE Trans. Microw. Theory Techn., vol. 60, no. 12, pp. 3908-3918, Dec. 2012.

[16] R. Rodríguez-Berral, F. Mesa, and F. Medina, "Analytical multimodal network approach for 2-D arrays of planar patches/apertures embedded in a layered medium," IEEE Trans. Antennas Propag., vol. 63, no. 5, pp. 1969-1984, May 2015.

[17] F. Mesa, M. García-Vigueras, F. Medina, R. Rodríguez-Berral, and J. R. Mosig, "Circuit-model analysis of frequency selective surfaces with scatterers of arbitrary geometry," IEEE Antennas Wireless Propag. Lett., vol. 14, pp. 135-138, Jan. 2015.

[18] S. R. Rengarajan, "Choice of basis functions for accurate characterization of infinite array of microstrip reflectarray elements," IEEE Antennas Wireless Propag. Lett., vol. 4, pp. 47-50, Jun. 2005.
[19] R. F. Harrington and J. R. Mautz, "Theory of characteristic modes for conducting bodies," IEEE Trans. Antennas Propag., vol. AP-19, no. 9, pp. 622-628, Sep. 1971.

[20] M. Cabedo-Fabres, E. Antonino-Daviu, A. Valero-Nogueira, and M. Ferrando-Bataller, "The theory of characteristic modes revisited: A contribution to the design of antennas for modern applications," IEEE Antennas Propag. Mag., vol. 49, no. 5, pp. 52-68, Oct. 2007.

[21] R. Dubrovka, J. Vazquez, C. Parini, and D. Moore, "Equivalent circuit method for analysis and synthesis of frequency selective surfaces," IEE Proc. H Microw. Antennas Propag., vol. 153, no. 3, pp. 213-220, Mar. 2006.

[22] S. Monni, G. Gerini, A. Neto, and A. G. Tijhuis, "Multi-mode equivalent networks for the design and analysis of frequency selective surfaces," IEEE Trans. Antennas Propag., vol. 55, no. 10, pp. 2824-2835, Oct. 2007.

[23] D. M. Pozar, Microwave Enginnering, 3rd ed. Hoboken, NJ, USA: Wiley, 2005.

[24] ANSYS High Frequency Structure Simulator (HFSS) [Online]. Available: http://www.ansys.com/Products/Electronics/ANSYS-HFSS, accessed on Feb. 2016.

[25] S. Maci, M. Caiazzo, A. Cucini, and M. Casaletti, "A pole-zero matching method for EBG surfaces composed of a dipole FSS printed on a grounded dielectric slab," IEEE Trans. Antennas Propag., vol. 53, no. 1, pp. 70-81, Jan. 2005. 